\title{
Spotlights on Recent JACS Publications
}

\section{nO CELLS REQUIRED: TACKLING TRICKY PROTEINS WITH METHYL LABELING}

Solving the structure of proteins can provide insight into biological function and aid in drug development. For proteins that resist crystallization, nuclear magnetic resonance (NMR) spectroscopy provides an alternate route to structural characterization. However, for certain proteins, it is difficult to obtain adequate quantities for NMR. Moreover, studying large proteins and membrane proteins by NMR is often hampered by broad signals and overcrowded spectra. To circumvent these obstacles, Rasmus Linser, Gerhard Wagner, and colleagues have developed a method to selectively label eukaryotic membrane proteins in a cell-free expression system (DOI: 10.1021/ ja504791j).

In cell-free expression, researchers mix together enzymes, nucleic acids, amino acids, and other cellular components that synthesize proteins. By incorporating amino acids with NMRactive ${ }^{13} \mathrm{C}$ and ${ }^{1} \mathrm{H}$ in methyl groups only, the team builds proteins that produce less crowded NMR spectra. To make such methyl-labeled amino acids, the researchers grow bacteria on a diet of metabolites containing ${ }^{13} \mathrm{C}$ and ${ }^{1} \mathrm{H}$ in specific locations. They express, purify, and digest a membrane protein to extract its amino acids, which contain the desired methyllabeling pattern. These amino acids are then used as ingredients in the cell-free expression of an integral membrane protein. The resulting NMR spectra demonstrate successful methyl labeling and offer a starting point to structural studies.

Erika Gebel Berg, Ph.D.

\section{SPEEDY DIELS-ALDER: A LITTLE LI+ GOES A LONG WAY}

Even though it was discovered nearly a century ago, the DielsAlder [4+2] cycloaddition reaction is still utilized extensively in modern synthetic chemistry. Now, a new report from Ken Kokubo, Yutaka Matsuo, and co-workers describes a way to dramatically increase the rate of this common pericyclic reaction under certain conditions (DOI: 10.1021/ja505952y).

One well-known approach for speeding up a Diels-Alder reaction involves Lewis acid catalysis, which requires that the dienophile bear a heteroatom to serve as a coordination site. But the addition of a Lewis acid catalyst introduces steric effects, which makes it impossible to determine the exact contribution of the electronic effects to the rate increase. In order to tease apart these contributions, the team compares [60] fullerene $\left(\mathrm{C}_{60}\right)$ with its $\mathrm{Li}^{+}$-encapsulated counterpart $\left(\mathrm{Li}^{+} @\right.$ $\mathrm{C}_{60}$ ) on the basis of each molecule's ability to undergo a cycloaddition reaction with 1,3-cyclohexadiene.

Because the Lewis acid, $\mathrm{Li}^{+}$, is contained inside the soccerball-shaped molecule, its presence does not affect the molecule's size, so any observed changes in reaction rate can be attributed fully to electronic effects. In this first-of-its-kind study, the team determines that the entrapped $\mathrm{Li}^{+}$causes the reaction to proceed 2400 times faster, a finding confirmed by both experimental and theoretical studies.

Christine Herman, Ph.D.

\section{COLOR-CHANGING SHAPE-SHIFTER AS FUTURE SMART MATERIAL}

Marisa Kozlowski and colleagues have synthesized a benzophenazine that changes color reversibly when crushed, with potential applications in a variety of smart materials (DOI: 10.1021/ja506137j).

Phenazines are redox-active compounds with multiple rings that have been used as dyes, sensors, and fluorescent probes. Kozlowski and colleagues noticed that one of the phenazines they synthesized, a tetrachlorinated axial chiral bisbenzophenazine, changes color when it is crushed between glass slides. In exploring what causes this mechanochromic activity, they find that the color change is reversed when the compound is exposed to an alkane solvent. They hypothesize that shearing disrupts the crystalline form of the compound to yield an amorphous form. Using X-ray crystallography, they determine that weak $\mathrm{O}-\mathrm{H} \cdots \mathrm{Cl}-\mathrm{C}$ hydrogen bonds hold the crystalline form together, creating pores for alkanes to bind.

The authors propose that this and similar phenazines could be used to create smart materials for a variety of uses. For instance, they could be added to plastics as a color-based indicator of stress to the material. The authors are also exploring using the molecules to develop nonmetal devices for rewritable data storage.

Deirdre Lockwood, Ph.D.

\section{THE POSITIVE AND NEGATIVE OF PROBING PEPTIDOGLYCAN}

Daniel Kahne, Suzanne Walker, and co-workers devise a new method for exploring the structure of the bacterial cell wall, and thereby develop a technology that could facilitate the design of better antibiotics (DOI: 10.1021/ja505668f). The cell wall, which protects bacteria from the dangers of the outside world, is composed of peptidoglycan, an intricate web of peptides, carbohydrates, and lipids.

Peptidoglycan is fabricated from a lipid-linked disaccharide pentapeptide called Lipid II. Probing a minor inherent difference in the structure of Lipid II in the cell wall of the Gram-negative bacteria Escherichia coli versus that in the Grampositive bacteria Bacillus subtilis, the authors elucidate functional differences in penicillin-binding proteins, the enzymes that construct peptidoglycan within the different bacteria types. Based on their findings, they create a novel fluorescent probe that enables highly efficient labeling of the cell wall of B. subtilis, a valuable tool for future studies.

The insights into the bacterial cell wall gained in this study illuminate the profound effect that seemingly subtle structural differences can have on the evolution and function of diverse bacterial species. Continued delineation of the structural and functional consequences of these differences offers opportunities for the development of novel biotechnological and therapeutic applications.

Eva J. Gordon, Ph.D.

Published: August 5, 2014 